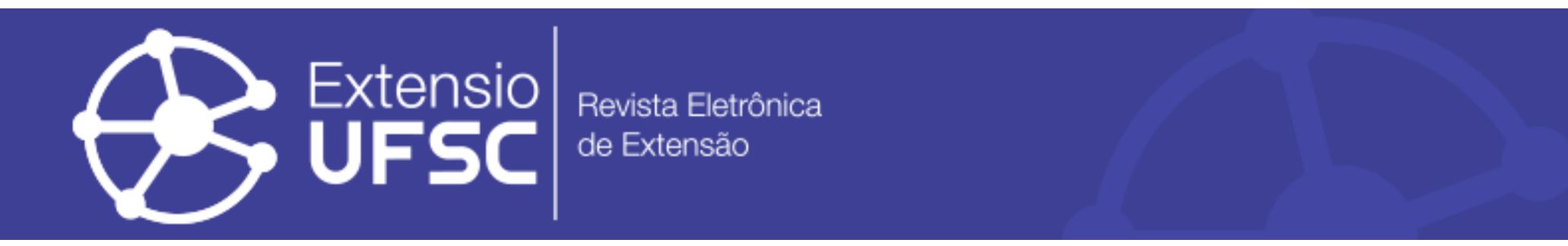

\title{
ESTÁGIO CURRICULAR SUPERVISIONADO EM UMA ESTRATÉGIA DE SAÚDE DA FAMÍLIA: UM RELATO DE EXPERIÊNCIA ACADÊMICO
}

\author{
Alessandra Schmidt \\ Universidade Federal do Pampa \\ alessandraschmidt1988@gmail.com \\ Bruna Stamm \\ Universidade Federal do Pampa \\ bruna-stamm@hotmail.com
}

\author{
Juliana Zubiaurre dos Santos \\ Prefeitura Municipal de Uruguaiana \\ juzubiaurre@gmail.com \\ Lilian Stumm \\ Prefeitura Municipal de Uruguaiana \\ lstumm132593@gmail.com \\ Maria Eduarda Deitos Vasquez \\ Universidade Federal do Pampa \\ maria.eduardadeitos@gmail.com
}

\section{Resumo}

O componente Estágio Curricular Supervisionado I (ECS I) da Universidade Federal do Pampa (UNIPAMPA), de caráter obrigatório, é cursado pelo discente do $9^{\circ}$ semestre de Graduação em Enfermagem. Este recebe a supervisão técnica de um enfermeiro do serviço de saúde, de um docente orientador e um supervisor de estágio. O estudo tem como objetivo descrever a experiência vivenciada pela acadêmica durante estágio na Estratégia de Saúde da Família (ESF). Trata-se de um relato de experiência, que descreve duas atividades do plano de trabalho na Dimensão Educação em Saúde, durante o componente curricular ECS I, nos meses de março e abril de 2018. As atividades foram: sala de espera sobre o tema colesterol e grupo de gestantes com participação da Residência Multiprofissional em Saúde Coletiva com ênfase no aleitamento materno. A experiência teve grande relevância, proporcionando elaborar e intervir com um plano de trabalho, correlacionar teoria e prática, além de criar vínculo com as equipes da ESF e população.

Palavras-chave: Enfermagem. Estratégia de Saúde da Família. Educação em Saúde.

\section{CURRICULAR STAGE SUPERVISED IN A FAMILY HEALTH STRATEGY: A REPORT OF ACADEMIC EXPERIENCE}

\begin{abstract}
The Curricular Component Supervised Internship I (ECS I) of the Federal University of Pampa (UNIPAMPA), which is compulsory, is taken by the student of the 9th semester of Undergraduate Nursing. The latter receives technical supervision from a health care nurse, a counselor, and an internship supervisor. The study aims to describe the experience lived by the academic during the internship in the Family Health Strategy (FHS). This is an experience report that describes two activities of the work plan in the Health Education Dimension during ECS I in the months of March and April 2018. The activities were: waiting room on the topic of cholesterol and group of pregnant women with participation of the Multiprofessional Residency in Public Health with emphasis on breastfeeding. The experience was highly relevant, providing elaboration and intervention with a work plan, correlating theory and practice, and creating a link with the FHS and population teams.
\end{abstract}

Keywords: Nursing. Strategy Health of the Family. Education and Health.

\section{ESTUDIO CURRICULAR SUPERVISADO EN UNA ESTRATEGIA DE SALUD DE LA FAMILIA: UN RELATO DE EXPERIENCIA ACADÉMICO}

\footnotetext{
Resumen

La Etapa Curricular Supervisionado I (ECS I) de la Universidad Federal de Pampa (UNIPAMPA), de carácter obligatorio, es cursado por el estudiante del $9^{\circ}$ semestre de Graduación en Enfermería. Este recibe la supervisión técnica de un enfermero del servicio de salud, de un docente orientador y un supervisor de prácticas. El estudio tiene como objetivo describir la experiencia vivenciada por la académica durante pasantía en la Estrategia de Salud de la Familia (ESF). Se trata de un relato de experiencia, que describe dos actividades del plan de trabajo en la Dimensión Educación en Salud, durante el ECS I, en los meses de marzo y abril de 2018. Las actividades fueron: sala de espera sobre el tema colesterol y grupo de gestantes con participación de la Residencia Multiprofesional en Salud Colectiva con énfasis en la lactancia materna. La experiencia tuvo gran relevancia, proporcionando elaborar e intervenir con un plan de trabajo, correlacionar teoría y práctica, además de crear vínculo con los equipos de la ESF y la población.

Palavras clave: La Enfermería. Estratégia Saúde de la Família. La Educación en la Salud.
} 
Estágio curricular supervisionado em uma estratégia de saúde da família: um relato de experiência acadêmico

\section{INTRODUÇÃO}

O Ministério da Saúde (MS) institui em 1994 o Programa de Saúde da Família (PSF), que mais tarde no ano de 2006 passou a ser chamado de Estratégia Saúde da Família (ESF), que tem como principal objetivo a reorganização do modelo assistencial do Sistema Único de Saúde (SUS). É considerada uma estratégia de expansão, qualificação e consolidação da Atenção Básica (AB), com o propósito de reorientar o processo de trabalho e aprofundar as diretrizes e fundamentos da mesma, assim como aumentar a resolutividade e servir de grande impacto para as coletividades (BRASIL, 2011).

A ESF caracteriza-se pela promoção da saúde de maneira territorializada, aumentando o acesso da população aos serviços de saúde, compondo-se de equipes multiprofissionais responsáveis pelo planejamento de intervenções conforme as necessidades dos indivíduos da comunidade (BRASIL, 2012). O seguinte processo de trabalho permite o compromisso e a corresponsabiblidade da equipe com o usuário, bem como a articulação entre os demais serviços de saúde que englobam o modelo assistencial (BRASIL, 2001).

As equipes multiprofissionais das ESF devem constituir de um médico generalista, um enfermeiro, um técnico ou auxiliar de enfermagem, quatro a seis Agentes Comunitários de Saúde (ACS), um odontólogo e um técnico em saúde bucal. Ademais, visam conhecer a área de abrangência, ampliando o vínculo com a comunidade, a qualidade e o acesso aos serviços prestados, bem como elaborar ações estratégicas e intervenções que desencadeiem melhorias na saúde da população. Assumem áreas geográficas definidas, com populações adstritas, contendo no máximo até 4.000 pessoas (BRASIL, 2012).

O enfermeiro como integrante da equipe de saúde na ESF tem como atribuições o planejamento, a coordenação, o gerenciamento, a execução e a avaliação das ações prestadas (BRASIL, 2012). Também coordena o processo de trabalho das equipes de saúde e cabe a ele planejar e aplicar quatro atividades essenciais: de assistência, de gerência, de educação e pesquisa, que ocorrem de maneira simultânea no seu cotidiano laboral (SPAGNUOLO et al., 2012).

Uma das principais ações a serem realizadas pelo enfermeiro na $A B$ é a educação em saúde, que pode ser desenvolvida com a própria equipe ou durante uma consulta de enfermagem, na sala da espera, em grupos terapêuticos, nas Visitas Domiciliares (VD) e atividades nas escolas (NASCIMENTO; NASCIMENTO, 2005).

Neste sentido, faz-se necessário que o enfermeiro receba um ensino de qualidade e saiba aplicar suas reais atribuições. Atividades de estágio apresentam uma ferramenta de aproximação entre a academia e os serviços, uma vez que emprega conhecimentos, habilidades e atitudes 
Estágio curricular supervisionado em uma estratégia de saúde da família: um relato de experiência acadêmico

profissionais apreendidos pelo estudante, fortalecendo suas competências por meio dos processos de trabalho das instituições de saúde. O componente Estágio Curricular Supervisionado (ECS) tem por objetivo proporcionar ao estudante a articulação da teoria e da prática em um processo de formação participativo, permeado pela interlocução entre o ensinar e o aprender em ambientes extramuros, com a participação ativa de profissionais da área de formação, universidade e comunidade (MARRAN; LIMA; BAGNATO, 2015).

Um estudo de revisão nacional analisou as contribuições do ECS na formação em enfermagem no Brasil, e identificou, dentre as publicações, que este oportuniza ao estudante caminhos e cenários de atuação capazes de edificar o exercício profissional. Por meio da práxis transformadora, o ECS fomenta a atuação segura e de qualidade, sedimenta competências profissionais e segurança para a realização do papel profissional no mercado de trabalho, e auxilia na reflexão crítica sobre a prática profissional e das instituições de saúde (ESTEVES; CUNHA; BOHOMOL; NEGRI, 2018).

Diante do exposto, o objetivo do presente relato é descrever as experiências de uma acadêmica de enfermagem acerca das atividades da dimensão educação em saúde, vivenciadas durante o componente ECS I.

\section{MATERIAIS E MÉTODOS}

Trata-se de um relato de experiência que descreve a vivência de uma acadêmica do curso de Graduação em Enfermagem da Universidade Federal do Pampa (UNIPAMPA) pelo componente curricular ECS realizado no período de março a abril de 2018, em uma ESF de um município da fronteira oeste do Rio Grande do Sul/Brasil.

O serviço conta com duas equipes de saúde, que partilham da mesma estrutura física e atendem a população de duas áreas distintas. Cada qual, constituída por um médico clínico geral, uma enfermeira, dois técnicos de enfermagem, quatro ACS e uma auxiliar de saúde bucal (atualmente não há odontólogo na equipe). Também há um terceiro médico clínico geral atendendo por contrato, uma nutricionista, um educador físico (integrantes do Núcleo Ampliado de Saúde da Família e Atenção Básica NASF-AB) e no presente ano conta com a atuação da Equipe Multiprofissional de Residentes em Saúde Coletiva da UNIPAMPA.

A população total assistida pela ESF é, atualmente, de 7.812 pessoas, sendo que 3.746 pertencem à equipe 01 e 4.066 à equipe 02, no entanto, das 13 micro-áreas pertencentes, sete delas estão descobertas e seis cobertas. O serviço funciona das 7:30 hs às 11:30 hs e das 13:30 hs até as 17:30 hs de segundas a sextas-feiras. 
Estágio curricular supervisionado em uma estratégia de saúde da família: um relato de experiência acadêmico

O referido componente ECS I, de caráter obrigatório ofertado no $9^{\circ}$ semestre de Graduação em Enfermagem possibilita o desenvolvimento de ações de enfermagem sob a supervisão técnica direta do enfermeiro do serviço de saúde, de um docente/enfermeiro orientador e um supervisor/enfermeiro de estágio (Técnico Administrativo em Educação TAE), vinculados ao curso de enfermagem, a fim de oportunizar a integração do acadêmico junto às instituições de saúde, por meio da realização de atividades específicas do profissional enfermeiro no âmbito da $\mathrm{AB}^{1}$.

O componente ECS I foi desenvolvido em 29 dias, com oito horas diárias de atividade, totalizando 230 horas tendo como cenário de prática uma ESF urbana. Diversas foram as atividades realizadas pela acadêmica durante o período de estágio, dentre elas: classificação de risco na triagem; avaliação de lesões de pele (necessidade de sutura); VD e medidas de conforto; consulta puerperal (mãe/bebê); realização e diagnóstico dos testes rápidos (Sífilis; HIV; Hepatite B e C); assistência ao pré-natal de risco habitual; coleta de exame citopatológico e exame clínico das mamas; prescrição de medicamentos (incumbência do enfermeiro conforme protocolo municipal de saúde para mulheres em período reprodutivo e pré-natal de risco habitual); encaminhamentos para a equipe de residência da unidade; Sistematização da Assistência de Enfermagem (SAE); consulta ao adolescente; ações de educação em saúde para a população: indivíduos crônicos, gestantes; participação nos debates de estudos de caso da Residência Multiprofissional.

Como proposta avaliativa do componente ECS I foi elaborado, implementado e avaliado um plano de trabalho, realizado pela própria acadêmica com orientação do docente/enfermeiro supervisor. Este contemplou ações relacionadas às dimensões de assistência, gerenciamento, controle social e educação em saúde, com o intuito de resgatar o conhecimento adquirido ao longo da graduação e atender as particularidades e necessidades da unidade de saúde e da população assistida. O plano foi executado a partir da elaboração de objetivos, métodos, intervenções e avaliações para cada atividade proposta nas dimensões. Em decorrência da ampliação da proposta do plano, o presente abordará àquelas elencadas na dimensão educação em saúde, referentes às atividades de sala de espera e grupo de gestantes realizadas na ESF. Informações quanto à organização, operacionalizações, metodologia e participantes serão detalhadas nos resultados e análises do presente artigo.

Ressalta-se que o desenvolvimento das atividades propostas no plano de trabalho foram ações que respeitaram os direitos éticos, de sigilo, de privacidade e de autonomia dos usuários

1 Plano de ensino do componente curricular Estágio Curricular Supervisionado I - Curso de Graduação em Enfermagem UNIPAMPA. Uruguaiana. 2018 (Digitado). 
Estágio curricular supervisionado em uma estratégia de saúde da família: um relato de experiência acadêmico

participantes, uma vez que possuíram supervisão do enfermeiro responsável da ESF e caracterizaram-se como iniciativas vinculadas ao serviço.

\section{RESULTADOS E ANÁLISES}

Durante a realização do ECS I obteve-se a oportunidade de vivenciar a rotina de assistência da ESF, acompanhar além das enfermeiras, os demais profissionais nas suas atividades e exercer o papel da enfermeira na unidade, colocando em prática o plano de trabalho.

Na primeira semana, foi realizado o diagnóstico situacional, no qual se pode conhecer a unidade de saúde; identificar as prioridades por meio do levantamento de dados; detectar a existência de limitações por parte das equipes; caracterizar o território, a população de abrangência do serviço e a clientela; reconhecer as potencialidades das equipes e por fim elaborar ações necessárias para o plano de trabalho a serem implementadas ao longo do estágio.

Um estudo realizado com médicos vinculados ao "Programa Mais Médicos" que concluíram a especialização no ano de 2015 em Saúde da Família pela Universidade Federal de Pelotas-RS, no modo à distância, destacaram que o diagnóstico situacional é a base fundamental para o planejamento estratégico de intervenções, com o objetivo de elencar problemas existentes nos serviços de saúde, o déficit na oferta e as reais necessidades da comunidade. A partir dele é elaborado o plano de ação, que vai desde o cronograma de atividades até a avaliação dessas ações e dentre as mais realizadas com a comunidade e profissionais encontram-se as de educação em saúde (THUMÉ et al., 2016).

O levantamento das informações se deu por meio da observação da acadêmica quanto ao funcionamento da unidade, caracterização da clientela e as atividades exercidas por cada profissional, além do acompanhamento e diálogo constante com as enfermeiras, no que se referiu ao processo de trabalho gerencial e assistencial. Com isso, foi possível constatar que a demanda significativa da unidade é a saúde da mulher de maneira integral, nas diversas fases de vida; idosos que vivem com problemas crônicos de saúde e monitoramento da caderneta de vacinação infantil.

A atividade "sala de espera" elaborada a partir da dimensão educação em saúde, foi realizada de forma coletiva com dois grupos diferentes de usuários que aguardavam atendimento na ESF. No decorrer da ação que durou em média de 15 minutos, houve a demonstração lúdica de modelos confeccionados em E.V.A imitando artérias, para ilustrar o tema "colesterol”, entrega de folders explicativos e breve discussão sobre o assunto a fim de sanar dúvidas diversas. Nesta foi abordado o tema colesterol, por meio de ações educativas de prevenção de doenças vasculares 
Estágio curricular supervisionado em uma estratégia de saúde da família: um relato de experiência acadêmico

e promoção da saúde, almejando a redução do número de indivíduos com o colesterol acima dos níveis indicados.

O significado de educação em saúde é um conceito amplo baseado na promoção da saúde, que abarca a participação de toda a população no âmbito da sua vida cotidiana e não apenas das pessoas sob o risco de adoecer. Refere-se à questão mantenedora de um estado positivo de saúde, que envolve aspectos físicos, ambientais e psicossociais (CARDOSO et al., 2016). É uma ferramenta que incita o auto cuidado do indivíduo, família e comunidade, promovendo reflexões para a modificação de atitudes e condutas (ROECKER; NUNES; MARCON, 2013).

Um relato de experiência sobre atividade desenvolvida em âmbito escolar por um acadêmico durante o ECS de uma universidade de Sete Lagoas-MG revela a importância de ações de educação em saúde no local de vivência dos adolescentes a fim de compartilharem problemas comuns, trocas de experiência e oportunizar espaços para análise da situação atual de saúde e projeção desta para o futuro. A atividade de educação em saúde buscou aproximar o público alvo do serviço de saúde por meio de discussões referente ao uso de drogas lícitas e ilícitas, Doenças Sexualmente Transmissíveis (DST’s), higiene e desnutrição de uma maneira diferenciada do que a mera transmissão de informações, a fim de auxiliar os sujeitos na corresponsabilização pela própria saúde. Tal atividade é uma das responsabilidades do enfermeiro e deve ser uma prática cotidiana da ESF, com colaboração da equipe multidisciplinar em saúde (SALUM; MONTEIRO, 2015).

A atividade de sala de espera foi realizada com o apoio de dois ACS e teve como tema “alterações lipídicas: o colesterol”, na qual estavam presentes usuários de variadas faixas etárias e motivos da busca pelo atendimento. Num primeiro momento, a acadêmica explanou sobre o que é o colesterol, a formação de trombos, a diferença entre o colesterol associado à Lipoproteína de Alta Densidade (HDL) e o colesterol associado à Lipoproteína de Baixa Densidade (LDL). Para melhor entendimento houve a demonstração lúdica com modelos confeccionados em papel E.V.A nos quais replicavam as artérias e os trombos formados em decorrências de alterações lipídicas no organismo.

No segundo momento, as ACS esclareceram aos usuários quanto à importância de uma alimentação saudável, da redução de peso, da prática de exercícios físicos, da diminuição no consumo de bebidas alcoólicas e tabaco, além de reforçar a informação de que a ESF tem atendimento nutricional e educação física que podem auxiliar no processo de reeducação alimentar e exercícios. Por último, os indivíduos foram estimulados a relatar experiências quanto 


\section{Estágio curricular supervisionado em uma estratégia de saúde da família: um relato de}

experiência acadêmico

ao tema colesterol, oportunizou-se um momento para esclarecimento de dúvidas e foram entregues folders explicativos. Esta atividade teve duração de 15 minutos, aproximadamente.

Em um estudo que avaliou o risco de doença cardiovascular de 71 pacientes que vivem com Doença Renal Crônica (DRC) e que frequentam um Centro de Hemodiálise em Belém-PA, a maioria não praticava atividade física. Como resultado da pesquisa, identificou-se que quase a metade dos indivíduos apresentou risco para doença cardiovascular, com predomínio do sexo masculino $(77,42 \%)$, grupo no qual demora em procurar atendimento no serviço de saúde e por vezes o diagnóstico de alguma patologia acaba sendo tardio (MORAES et al., 2017). Tendo em vista os altos índices de pessoas com alterações de TG/HDLc, e com base no estudo, é que foi planejado o tema da sala de espera a fim de conscientizar a população quanto os cuidados com a saúde.

A adesão da comunidade em grupos educativos ofertados pelo serviço de saúde, no caso as ESF, ainda é insatisfatória, no entanto, as salas de espera transformaram-se em uma estratégia simples e potencialmente efetiva para abordar questões relacionadas à promoção e prevenção da saúde. Atividades deste cunho favorecem a participação de usuários dos quais normalmente não aderem a esse tipo de atividade em grupo, como é o caso do público dos adolescentes e dos homens. Por outro lado, limitações como o tempo reduzido e o risco de dispersão dos participantes após passarem pelo atendimento, podem tornar a atividade educativa falha ou insuficiente (SILVA et al., 2017).

Um estudo realizado no sudeste de Michigan (EUA) revelou que uma breve intervenção baseada na sala de espera foi associada ao aumento do uso de um programa de incentivo alimentar saudável, denominado Double Up Food Bucks (DUFB), no qual adultos inscritos em um centro de saúde localizado em uma região de baixa renda incentiva a população a comprar em mercados de agricultores, aumentando o consumo de verduras e frutas, tornando a alimentação mais saudável (COHEN et al., 2017).

Acredita-se que a atividade de sala de espera é indispensável e as informações nela adquiridas sejam compartilhadas na comunidade. No entanto, não é possível visualizar resultados de maneira imediata, para tanto se faz necessário que a ESF continue a desenvolver este tipo de ação a fim de fortalecer e relembrar assuntos, utilizando-se de pouco tempo e de estratégias lúdicas para melhor entendimento.

A segunda atividade realizada na dimensão de educação em saúde do plano de trabalho foi uma ação integrada da acadêmica com a equipe da Residência Multiprofissional em Saúde Coletiva, composta por uma enfermeira, uma nutricionista, uma educadora física e duas fisioterapeutas. Esta ocorreu durante o encontro mensal com um grupo de quatro gestantes 
Estágio curricular supervisionado em uma estratégia de saúde da família: um relato de experiência acadêmico

pertencentes a ESF, e teve como objetivo abordar ações multidisciplinares acerca do aleitamento materno. A acadêmica de enfermagem abordou questões relacionadas ao preparo das mamas e pega correta do $\mathrm{RN}$; as fisioterapeutas residentes trabalharam a respeito de exercícios para períneo, a educadora física residente demonstrou exercícios de relaxamento e a nutricionista residente apresentou os diversos benefícios do aleitamento materno exclusivo até os seis meses de idade do RN.

Para o desenvolvimento do grupo de gestantes foram utilizados diversos recursos: no primeiro momento houve uma breve explicação no formato de slides sobre a importância do aleitamento materno exclusivo, o seu benefício a fim de evitar complicações para a saúde do RN, aspectos afetivos que podem ser fortalecidos durante a mamada, anatomia da mama, tipos de leite (colostro; transição; maduro) além de dicas sobre a pega correta do seio. Na sequência, foram reproduzidas, cenas com o auxílio de uma boneca, acerca das posições adequadas para amamentação, orientações sobre cuidados com a mama antes e após amamentação, e utilizou-se também uma mama confeccionada artesanalmente de meia-calça para demonstração da pega correta do RN no seio materno, complementarmente ao uso de fotos ilustrativas.

As fisioterapeutas residentes indicaram dois vídeos dos quais foram reproduzidos para as gestantes a respeito da mecânica da mamada, que mostrou o movimento que o RN faz com a língua no seio para sucção do leite e o outro de como tirar o RN do seio para não machucá-lo; também foram entregues folders de apenas uma página, que continham figuras das diferentes posições indicadas para amamentação. O tempo de duração desta atividade foi de aproximadamente 50 minutos, acrescido ao tempo para esclarecimento de dúvidas pelas gestantes.

No período da gestação a mulher está vulnerável, exposta a múltiplas exigências, vivenciando um período de adaptação ou reorganização corporal, hormonal, familiar e social (FALCONE et al., 2005). A fim de ampará-las, o grupo de gestantes viabiliza o intercâmbio de experiências e conhecimentos, sendo considerada a melhor forma de promover a compreensão do processo de gestação. São discutidas informações sobre as diferentes vivências entre as mulheres e os profissionais de saúde (BRASIL, 2006).

Quanto ao aleitamento materno, o Ministério da Saúde (MS) recomenda que o mesmo deva ser exclusivo nos primeiros seis meses de vida, sendo este um alimento completo para o RN devido suas propriedades nutricionais e imunológicas que primeiramente diminuem o risco de mortalidade infantil, oferecem proteção contra possíveis infecções, doenças respiratórias, diarreias, riscos de obesidade e desenvolvimento de hipertensão e diabetes, bem como fortalece o crescimento e desenvolvimento saudável da criança (BRASIL, 2009). 
Estágio curricular supervisionado em uma estratégia de saúde da família: um relato de experiência acadêmico

Um estudo descritivo e exploratório do qual participaram 24 gestantes atendidas em uma ESF do município de Palmital do estado de São Paulo destaca a importância de a gestante receber orientações quanto ao aleitamento materno ainda na primeira consulta de pré-natal, e ser acompanhada por uma equipe multiprofissional de maneira individualizada ou em grupos de educação em saúde, com a finalidade de trabalhar questões como as vantagens, as possíveis dificuldades, a anatomia e a fisiologia e os mitos e verdades frente ao processo de amamentação (FERREIRA; GOMES; FRACOLLI, 2018).

Um relato de experiência sobre grupos de gestantes realizados por acadêmicos do curso de enfermagem da Universidade Estadual Vale do Acaraú (UVA), no município de Sobral-CE, revela que esse tipo de atividade é uma importante estratégia de educação em saúde e de responsabilidade do enfermeiro da AB. Propicia o fortalecimento de vínculo entre os profissionais de saúde e gestantes, além de direcionar ações para um grupo significativo, que influencia a vida das gestantes e contribui para a redução de riscos que possam afetar a vida da mulher e do feto. Durante o grupo é possível realizar ações de promoção à saúde, ao mesmo tempo em que as gestantes esclarecem dúvidas com o profissional, ouvem relatos das demais mulheres e são motivadas quanto à maternidade, mas, muitas vezes essa atenção se torna inviável no cotidiano dos profissionais devido à sobrecarga de trabalho da unidade básica (VASCONCELOS et al., 2017).

O resultado do estudo acima vai de encontro à realidade vivenciada no grupo de gestantes, onde não somente primigestas referiram suas dúvidas quanto aos assuntos relacionados à gravidez, mas também aquelas mulheres que já passaram por uma gravidez anteriormente. Com isso, percebe-se a implicação de ações de educação em saúde, a fim de compartilhar conhecimentos para a mulher e quando possível ao parceiro, com o objetivo de que a maternidade seja experienciada de maneira tranquila e prazerosa.

Ao final do grupo de gestantes foi distribuído para cada participante, um mini questionário com perguntas: $O$ que você achou sobre a interação entre os profissionais (residência multiprofissional e acadêmica de enfermagem) na condução do grupo? Para os próximos encontros qual(is) tema(s) seria(m) importante(s) ser(em) abordado(s)? Quanto à primeira questão todas as mulheres relataram suma importância a interação multiprofissional, porque ambas conseguiram esclarecer dúvidas diversas de forma direta com o profissional, cada qual na sua área. Solicitaram para os próximos encontros assuntos como a diferença entre parto vaginal e cesárea, indicações e contra-indicações de cada, cuidado com os seios, a forma correta de dar banho no RN e o desenvolvimento do bebê nos primeiros meses de vida. 
Estágio curricular supervisionado em uma estratégia de saúde da família: um relato de experiência acadêmico

Ao término do ECS I a acadêmica compartilhou com a equipe de profissionais e da residência multiprofissional os assuntos indicados pelas gestantes, com o intuito de continuidade da proposta.

As atividades de educação em saúde, como as realizadas durante o estágio devem ser ações freqüentes do enfermeiro junto à equipe de saúde e da comunidade do território. É uma importante ferramenta para transmissão de conhecimento, que almeja primeiramente a prevenção e promoção da saúde, por vezes, a reeducação de famílias quanto o viver com doenças crônicas, em busca da qualidade de vida. Além disso, é um momento que a população tem de esclarecer dúvidas, de criar vínculo, ações estas que propiciam melhora do acesso e da assistência de saúde prestada.

\section{CONSIDERAÇÕES FINAIS}

A experiência do componente ECS I foi de grande relevância para o crescimento da acadêmica e futura profissional, podendo correlacionar teórica e prática, superando dificuldades e aprimorando potencialidades. Além disso, vivenciou as reais atribuições da enfermeira de uma ESF e conseguiu, junto às elas e a docente supervisora, elaborar um plano de trabalho aplicável e com resultados impactantes à população do território. Esta experiência permitiu aquisição de habilidades e competências pela acadêmica, almejando futura atuação profissional como enfermeira, e autonomia para realizar atividades privativas e inerentes a atuação da enfermeira na $\mathrm{AB}$.

A atividade de sala de espera tem grande potencial para a realização de atividades de educação em saúde, mesmo que de forma pontual. Diferentemente do grupo de gestantes, em que o público alvo busca conhecimento e procura dicas para o bem estar na gravidez, faz com que usufruam ao máximo dos encontros.

Percebeu-se que as atividades de educação em saúde merecem maior atenção e precisam ser desenvolvidas com a comunidade constantemente, pois ainda vive-se a realidade que os indivíduos tendem o olhar para a saúde como ausência de doença, desacostumados a praticarem ações de prevenção e promoção e esta visão deve ser trabalhada com as comunidades e famílias, no âmbito das ESF's.

E por fim, relatar a experiência de uma acadêmica de enfermagem em ações de educação em saúde na $\mathrm{AB}$ tem relevância científica no sentido em que compartilha perspectivas de cuidado ampliadas e exitosas, possibilitando para a formação de enfermagem subsídios práticos, pautados na articulação com a teoria do cuidar. 
Estágio curricular supervisionado em uma estratégia de saúde da família: um relato de experiência acadêmico

\section{REFERÊNCIAS}

BRASIL. Ministério da Saúde. Secretária de Políticas Públicas. Guia Prático do Programa de Saúde da Família. Brasília: MS, 2001. Disponível em: http://dab.saude.gov.br/portaldab/biblioteca.php?conteudo=publicacoes/guia_pratico_saude_f amilia. Acesso em: 02 de maio de 2018.

BRASIL. Ministério da Saúde. Secretaria de Atenção a Saúde. Departamento de Ações Programáticas Estratégicas. Pré-natal e puerpério: atenção qualificada e humanizada: manual técnico. Brasília: Ministério da Saúde, 2006. 162p. Disponível em: http://bvsms.saude.gov.br/bvs/publicacoes/manual_pre_natal_puerperio_3ed.pdf. Acesso em: 03 de maio 2018.

BRASIL. Ministério da Saúde. Secretaria de Atenção à Saúde. Departamento de Atenção Básica. Saúde da criança: nutrição infantil: aleitamento materno e alimentação complementar. Brasília; 2009.2 Disponível em: http://bvsms.saude.gov.br/bvs/publicacoes/saude_crianca_nutricao_aleitamento_alimentacao.p df. Acesso em: 06 de maio de 2018.

BRASIL. Ministério da Saúde. Secretaria de Assistência à Saúde. Gabinete do Ministro. Política Nacional de Atenção Básica. Portaria no 2.488 de 21 de outubro de 2011. Brasília: 2011. Disponível em: http://bvsms.saude.gov.br/bvs/saudelegis/gm/2011/prt2488_21_10_2011.html. Acesso em: 04 de maio de 2018.

BRASIL. Ministério da Saúde. Secretaria de Atenção à Saúde. Departamento de Atenção Básica. Política Nacional de Atenção Básica. Brasília: MS, 2012. Disponível em: http://189.28.128.100/dab/docs/publicacoes/geral/pnab.pdf. Acesso em: 06 de maio de 2018.

COHEN, A. J. et al. Increasing use of a healthy food incentive: A waiting room intervention among low-income patients. American Journal of Preventive Medicine, v. 52, n. 2, p. 154-162, 2017. Disponível em: https://www.ajpmonline.org/article/S0749-3797(16)30582-7/fulltext. Acesso em: 06 de maio de 2018.

ESTEVES, L. S. F.; CUNHA, I. C. K. O.; BOHOMOL, E.; NEGRI, E. C. O estágio curricular supervisionado na graduação em enfermagem: revisão integrativa. Revista Brasileira de Enfermagem, v. 71 , n. suppl 4, p. 1842-53, 2018. Disponível em: http://www.scielo.br/pdf/reben/v71s4/pt_0034-7167-reben-71-s4-1740.pdf. Acesso em: 13 de fevereiro de 2019.

FALCONE, V. M. et al. Atuação multiprofissional e a saúde mental de gestantes. Revista de Saúde Pública [online], vol.39, n.4, p. 612-618, 2005. Disponível em: https://www.scielosp.org/scielo.php?pid=S0034-

89102005000400015\&script=sci_arttext\&tlng=ptpt. Acesso em: 07 de maio de 2018.

NASCIMENTO, M. S. et al. Oficinas pedagógicas: Construindo estratégias para a ação docenterelato de experiência. Saúde. com, v. 3, n. 1, 2016. Disponível em: http://www.uesb.br/revista/rsc/ojs/index.php/rsc/article/view/65/435. Acesso em: $11 \mathrm{de}$ maio de 2018. 
Estágio curricular supervisionado em uma estratégia de saúde da família: um relato de experiência acadêmico

FERREIRA, M. G. C.; GOMES, M. F. P.; FRACOLLI, L. A. Aleitamento Materno: Orientações Recebidas por Gestantes Acompanhadas pela Estratégia Saúde da Família. Revista de Atenção à Saúde, v. 16, n. 55, p. 36-41, 2018. Disponível em: http://seer.uscs.edu.br/index.php/revista_ciencias_saude/article/view/4888. Acesso em: 10 de maio de 2018.

MARRAN, A. L.; LIMA, P. G.; BAGNATO, M. H. S. As políticas educacionais e o estágio curricular supervisionado no curso de graduação em enfermagem. Revista Trabalho, Educação e Saúde, v.1, n. 1, p. 89-108, 2015. Disponível em: http://www.scielo.br/pdf/tes/v13n1/19817746-tes-1981-7746-sip00025.pdf. Acesso em: 13 de fevereiro de 2019.

MORAES, L. L. et al. Identificação de risco cardiovascular pela razão triglicerídeo/HDLcolesterol em pacientes com doença renal crônica em hemodiálise. Scientia Medica, v. 27, n. 3, p. 1, 2017. Disponível em: https://dialnet.unirioja.es/servlet/articulo?codigo=6137534. Acesso em: 11 de maio de 2018.

NASCIMENTO, M. S.; NASCIMENTO, M. A. A. Prática da enfermeira no Programa de Saúde da Família: a interface da vigilância da saúde versus as ações programáticas em saúde. Revista Ciência \& Saúde coletiva, v. 10, n. 2, p. 333-45, 2005. Disponível em: http://www.scielo.br/scielo.php?script=sci_arttext\&pid=S1413-81232005000200011. Acesso em: 14 de maio de 2018.

ROECKER, S.; NUNES, E. F. P. A.; MARCON, S. S. O trabalho educativo do enfermeiro na estratégia saúde da família. Texto \& Contexto em Enfermagem, v. 22, n. 1, p. 157-165, 2013. Disponível em: https://www.redalyc.org/pdf/714/71425827018.pdf. Acesso em: 12 de maio de 2018.

SALUM, G. B.; MONTEIRO, L. A. S. Educação em saúde para adolescentes na escola: um relato de experiência. Revista Mineira de Enfermagem, v. 19, n. 2, p. 246-257, 2015. Disponível em: http://www.reme.org.br/artigo/detalhes/1019. Acesso em: 05 de maio de 2018.

SILVA, E. F. et al. "Sala de espera": Cenário e estratégia de educação em saúde. JMPHC| Journal of Management \& Primary Health Care, v. 7, n. 1, p. 70-70, 2017. Disponível em: http://jmphc.com.br/jmphc/article/view/396/Sala\%20de\%20espera. Acesso em: 08 de maio de 2018.

SPAGNUOLO, R. S. et al. O enfermeiro e a estratégia saúde da família: desafios em coordenar a equipe multiprofissional. Ciência, Cuidado e Saúde, v. 11, n. 2, p. 226-34, 2012. Disponível em: http://www.periodicos.uem.br/ojs/index.php/CiencCuidSaude/article/view/10445/pdf.

Acesso em: 09 de maio de 2018.

THUMÉ, E. et al. Reflexões dos médicos sobre o processo pessoal de aprendizagem e os significados da especialização à distância em saúde da família. Revista Ciência \& Saúde Coletiva. v. 21, n. 9, p. 2807-2814, 2016. Disponível em: http://www.scielo.br/scielo.php?pid=S1413$81232016000902807 \&$ script=sci_abstract\&tlng=pt. Acesso em: 12 de maio de 2018.

VASCONCELOS, M. I. O. et al. Intervenção Educativa em Saúde com Grupo de Gestantes: Estudantes de Enfermagem em Ação Extensionista no Interior do Ceará. Expressa Extensão, v. 21, n. 2, p. 108-118, 2017. Disponível em:

https://periodicos.ufpel.edu.br/ojs2/index.php/expressaextensao/article/view/9248. Acesso em: 14 de maio de 2018. 
Estágio curricular supervisionado em uma estratégia de saúde da família: um relato de experiência acadêmico

Recebido em: 15/06/2018

Aceito em: 07/03/2019 